\title{
The Promotion Effect of Low-Molecular Hydroxyl Compounds on the Nano-Photoelectrocatalytic Degradation of Fulvic Acid and Mechanism
}

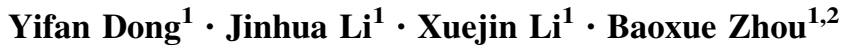

Received: 4 March 2016/Accepted: 29 March 2016/Published online: 4 May 2016

(C) The Author(s) 2016. This article is published with open access at Springerlink.com

\begin{abstract}
A significant promotion effect of low-molecular hydroxyl compounds (LMHCs) was found in the nano-photoelectrocatalytic (NPEC) degradation of fulvic acid (FA), which is a typical kind of humic acid existing widely in natural

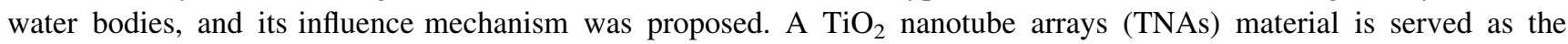
photoanode. Methanol, ethanediol, and glycerol were chosen as the representative of LMHCs in this study. The adsorption performance of organics on the surface of TNAs was investigated by using the instantaneous photocurrent value. The adsorption constants of FA, methanol, ethanediol, and glycerol were 43.44, 19.32, 7.00, and 1.30, respectively, which indicates that FA has the strongest adsorption property. The degradation performance of these organics and their mixture were observed in a thin-layer reactor. It shows that FA could hardly achieve exhausted mineralization alone, while LMHCs could be easily oxidized completely in the same condition. The degradation degree of FA, which is added LMHCs, improves significantly and the best promotion effect is achieved by glycerol. The promotion effect of LMHCs in the degradation of FA could be contributed to the formation of a tremendous amount of hydroxyl radicals in the NPEC process. The hydroxyl radicals could facilitate the complete degradation of both FA and its intermediate products. Among the chosen LMHCs, glycerol molecule which has three hydroxyls could generate the most hydroxyl radicals and contribute the best effective promotion. This work provides a new way to promote the NPEC degradation of FA and a direction to remove humus from polluted water.
\end{abstract}

\section{Graphical Abstract}

Jinhua Li

lijinhua@sjtu.edu.cn

$\triangle$ Baoxue Zhou

zhoubaoxue@sjtu.edu.cn

1 School of Environmental Science and Engineering, Shanghai Jiao Tong University, Shanghai 200240, People's Republic of China

2 Key Laboratory for Thin Film and Microfabrication of the Ministry of Education, Shanghai Jiao Tong University, Shanghai 200240, People's Republic of China
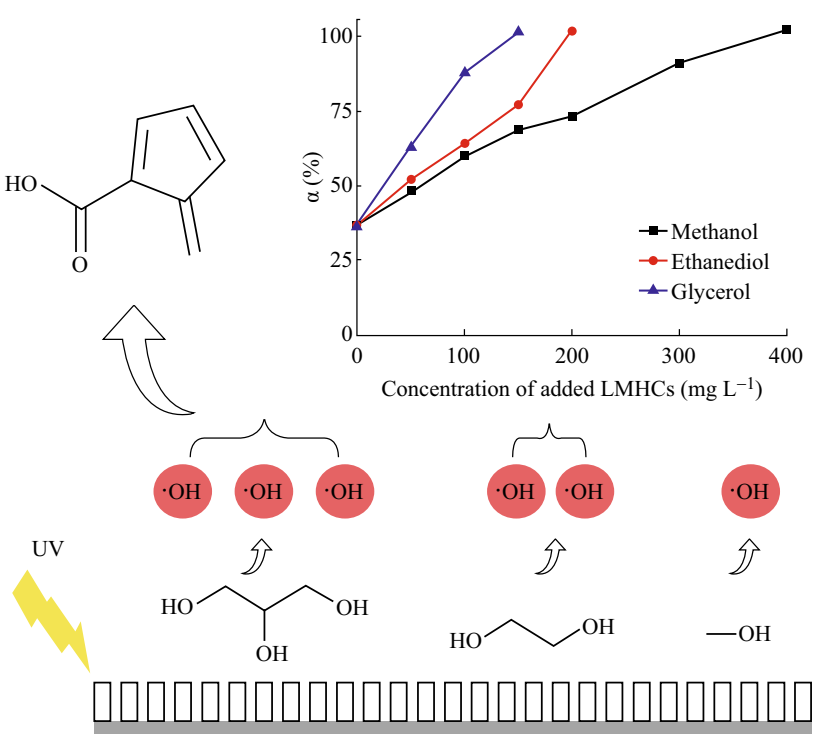
Keywords Fulvic acid · Nano-photoelectrocatalytic degradation $\cdot$ Promotion effect $\cdot$ Low-molecular hydroxyl compounds

\section{Introduction}

Fulvic acid (FA) is the main component of humus, which is a dominating natural organic matter $(\mathrm{NOM})$ in the water body $[1,2]$. Meanwhile, it is a refractory organic and also a major precursor of disinfection by-products (DBPs) which is formed in the process of chlorine disinfection in drinking water treatment. The DBPs are categorized as a kind of harmful materials which are carcinogenic, teratogenic, and mutagenic [3]. As a consequence, discovering a highly efficient and stable method to degrade FA is particularly important.

To remove FA from the water body, limited methods are found, which are enhanced coagulation, granular-activated carbon adsorption, and membrane filtration. Among them, the enhanced coagulation is the best way to control the amount of NOM. However, it is only effective to the mixture which includes large concentration of colloid humic acid and ineffective to the solution which has solute low molecular like FA in it $[4,5]$.

In recent years, to degrade a series of refractory organics, FA especially, the advanced oxidation technologies have become a research's hot spot, because it can generate hydroxyl radicals which have well-degradation ability. As an effective advanced oxidation technology, the photoelectrocatalytic (PEC) technology has many advantages, such as good oxidation ability, high organic matter removal efficiency, and no secondary pollution and so on. $\mathrm{TiO}_{2}$ nanotube arrays (TNAs) are chosen to be a photoanode material, which has larger specific surface area and more stable structure than other forms of $\mathrm{TiO}_{2}$ [6-9]. Meanwhile, it shows better catalytic properties and could degrade more kinds of refractory organic matter [10-17].

The PEC degradation of organics could not only be affected by the type of photocatalyst, the configuration of reactor, but also be affected by the reaction medium [1821]. For example, some chemical substances, such as tertiary butyl alcohol, phosphate, and carbonate could inhibit the hydroxyl radical's activity, so they could further inhibit the PEC degradation of organics [22].

The feasibility of PEC technology used in the degradation of FA is proved in the limited previous researches $[8,9]$. However, the reported method of FA degradation is inefficient and time-consuming, or the preparation of the catalytic material is very complex. In this work, in order to fast and accurately measure the degradation of organics and explore the degradation mechanism, an efficient catalytic material, TNAs, and a thin-layer PEC reactor were used. The PEC degradation of FA is under study, and a significant PEC promotion effect was found in the degradation of FA by adding low-molecular hydroxyl compounds (LMHCs) including methanol, ethanediol, and glycerol, which are widely existed in the water body and polluted water. The influence mechanism was proposed.

\section{Experiment}

\subsection{Material and Sample Preparation}

Unless specially indicated, all the reagents were analytical pure grade and were purchased from Sinopharm Chemical Reagent Company (Shanghai, China). All solutions were made up with high-purity de-ionized water $(18 \mathrm{M} \Omega)$ purified by a Milli-Q purification system (Millipore Corporation, Billerica, MA), and the supporting electrolyte in the samples is a $\mathrm{NaNO}_{3}$ solution.

\subsection{Preparation of the TNA Electrode}

The $\mathrm{TiO}_{2}$ nanotube electrodes were prepared according to the electrochemical anodic oxidation method which is reported in previous work [23]. The anode was titanium and the cathode was platinum. They were put into a mixture consisted of $1 \mathrm{~mol} \mathrm{~L}^{-1} \mathrm{NaF}, 1 \mathrm{~mol} \mathrm{~L}{ }^{-1} \mathrm{NaHSO}_{4}$, $0.2 \mathrm{~mol} \mathrm{~L}^{-1}$ trisodium citrate, and $\mathrm{NaOH}$ was added to adjust the $\mathrm{pH}$. The $\mathrm{TiO}_{2}$ nanotube electrodes would be formed in the solution under constant stirring for $6 \mathrm{~h}$ with an applied bias of $20 \mathrm{~V}$. Then, they were annealed in a laboratory muffle furnace at $500{ }^{\circ} \mathrm{C}$ lasting for $3 \mathrm{~h}$ to form anatase TNAs.

\subsection{The Characterization Method of TNA Electrode}

The surface morphology of TNAs working electrode was investigated by field emission scanning electron microscopy (Nova NanoSEM 450, FEI Company, USA) under a voltage of $5 \mathrm{kV}$. The crystalline phase of TNAs working electrode was identified by X-ray diffraction (XRD-6100, Shimadzu, Japan), using $\mathrm{Cu} K \alpha(\lambda>0.15406 \mathrm{~nm})$ radiation at $40 \mathrm{kV}$ and $30 \mathrm{~mA}$ at a scanning rate of $10^{\circ} \mathrm{min}^{-1}$ in the $2 \theta$ range from $10^{\circ}$ to $90^{\circ}$ at room temperature. The elements present in the TNAs electrode were identified by X-ray photoelectron spectroscopy (XPS, VG Microlab $310 \mathrm{~F}$, Al Ka radiation).

To explore this TNA's photoelectric properties, the characterization of TNAs working electrode was investigated and the electrolyte solution was oxidized under three 
degradation conditions: photocatalytic (PC), electrocatalytic (EC), and photoelectrocatalytic (PEC). The PEC condition was performed by UV illumination from a $365 \mathrm{~nm}$ ultraviolet LED with a $37.4 \mathrm{~mW} \mathrm{~cm}^{-2}$ light intensity as well as a $2 \mathrm{~V}$ bias voltage, which avoid the photoinduced electron and the hole from recombining. The $\mathrm{PC}$ and EC condition were performed without bias voltage or UV illumination, respectively.

\subsection{Reactor Used in the Experiment}

In this study, the reaction and degradation process were carried out in a thin-layer reactor. As shown in Fig. 1, the thin-layer reactor is a three-electrode system made up with six main sections: the TNAs working electrode, the platinum counter electrode, the saturated $\mathrm{Ag} / \mathrm{AgCl}$ reference electrode, the flow inlet, outlet, and a quartz window with a diameter of $1 \mathrm{~cm}$. Two polytetrafluoroethylene planks were adhered together to build a reaction cell. The thickness of the cell was controlled at most $0.1 \mathrm{~mm}$ to shorten the time of degradation and the distance of the mass transfer from the solution to the surface of electrode, and ensure the light transmittance of a $365 \mathrm{~nm}$ ultraviolet LED in the meanwhile. The potential and the current of the working electrode were controlled and monitored by an electrochemical workstation (CHI 610D, Chenghua, Shanghai) which was connected to the computer to record the photocurrent response signals.

\subsection{The Concentration Unit of the Organics and the Degradation Degree}

The concentration unit of organics in oxygen equivalent $\left(\mathrm{mg} \mathrm{L}^{-1}\right.$ ) was used in this study, since the transfer of each 4 mol electrons in the PEC degradation process is equivalent to the consumption of $1 \mathrm{~mol} \mathrm{O}_{2}$. This way will facilitate the evaluation of chemical reaction behavior of different organics as it loses the same amount of electrons [24].

The degradation degree $(\alpha)$ could be calculated according to Eq. 1, in which $Q_{\text {net }}$ was the measured value of the charge transfer quantity and $Q_{\mathrm{th}}$ was the theoretical

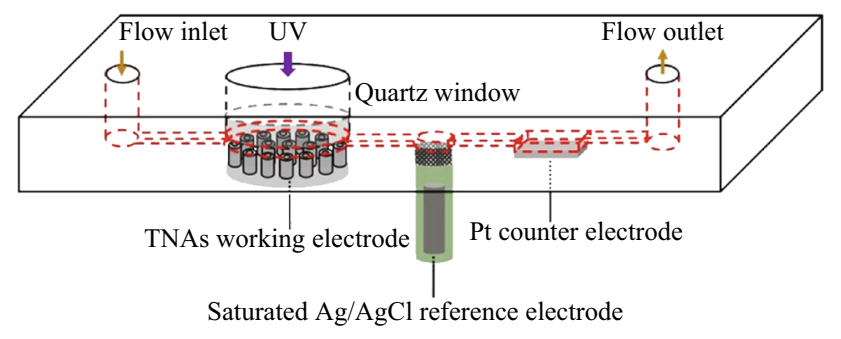

Fig. 1 The structure of the thin-layer reactor value of the charge transfer quantity in the PEC degradation process.

$\alpha=Q_{\text {net }} / Q_{\text {th }}$

$Q_{\text {net }}$ could be obtained by computing the integral area of the response signals shown in Fig. 2 according to Eq. 2.

$Q_{\text {net }}=\int I \mathrm{dt}$

$Q_{\text {th }}$ could be obtained by an exhausted PEC oxidation process of a standard substance, such as glucose [24, 25].

\section{Results and Discussion}

\subsection{Characterization of TNA Electrode}

The $I-t$ curves obtained from three degradation condition are shown in Fig. 3a. Both the current values of the PC and EC degradation are much smaller than the PEC degradation, so the degradations under PC and EC are ineffective, which illustrates the potential and UV light illumination work out a synergistic effect in the PEC oxidation process of the electrolyte solution (and as well as other matters, such as organics especially). The SEM of the TNAs shown in Fig. $3 b$ reveals that the nanotubes are highly ordered. The XRD patterns of TNAs electrode shown in Fig. 3c indicate that the sample possesses characteristic peaks for the anatase phase. In order to further investigate the chemical composition and oxidation state of $\mathrm{TiO}_{2}$ nanotube arrays, XPS measurements were performed. Figure 3d shows a typical XPS survey scan for the TNAs material over a large energy range at low resolution, which represents $\mathrm{Ti}^{4+}, \mathrm{O}^{2-}$ of $\mathrm{TiO}_{2}$ and some traces of carbon. These results further confirm the successful preparation of TNAs.

\subsection{The Adsorption Property}

As shown in Fig. 4a, in the beginning of the FA's PEC degradation, the initial instantaneous current value $\left(I_{0}\right)$ rises as the concentration of FA increases, which is related with its adsorption property [26].

According to the computer simulation, the relationship between the $I_{0}$ and the concentration of FA could fit with the following equation:

$$
\begin{aligned}
y= & 6.76 \times 10^{-5} \times 43.44 x /(1+43.44 x)+3.39 \\
& \times 10^{-5}\left(R^{2}=0.97\right)
\end{aligned}
$$

Equation 3 is similar to the Langmuir adsorption equation, which could appropriately describe the adsorption property of the solute on the interface of solid material in the solution $[27,28]$ : 


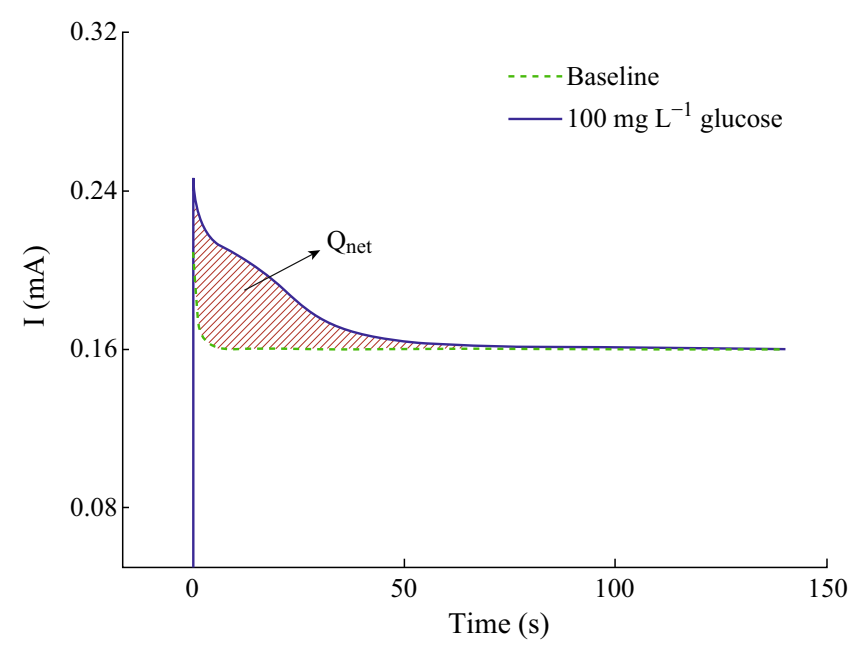

Fig. 2 The response signals in the glucose degradation

(a)

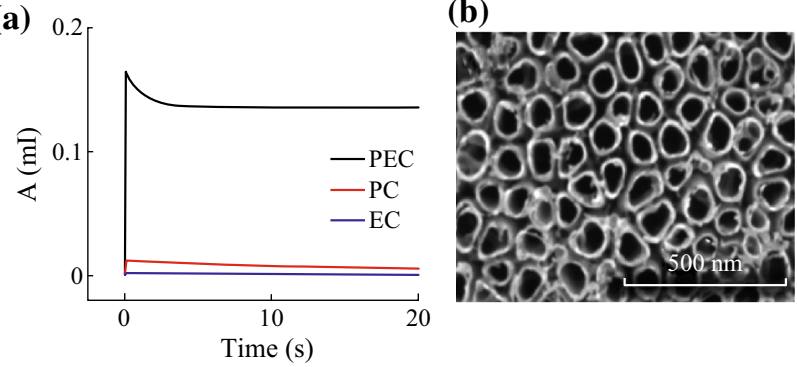

(c)

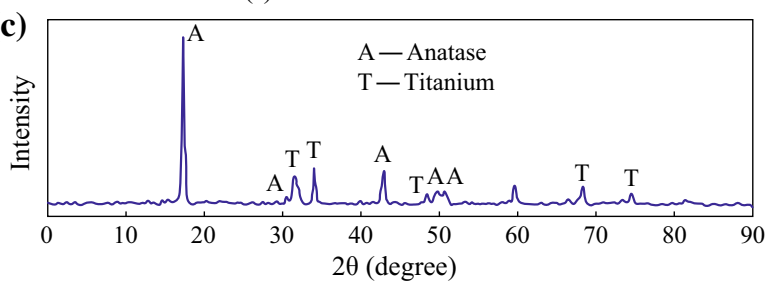

(d) $6000^{\mathrm{Ti}} \mathrm{LMM}$

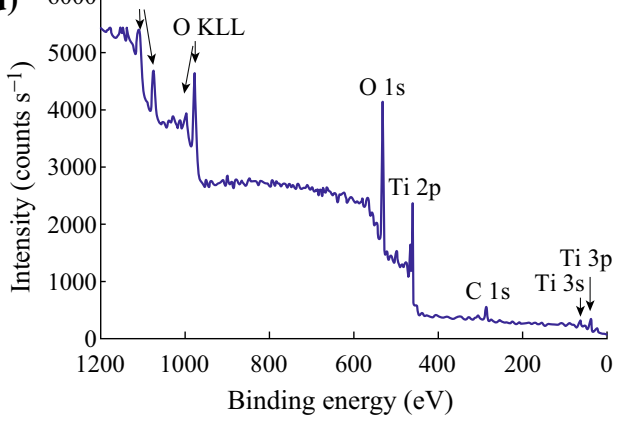

Fig. 3 The characterization of TNA electrode. a photoelectric property, b SEM, $\mathbf{c}$ XRD pattern, and d XPS of TNA electrode

$y=A \times B \times x /(1+B \times x)+C$

In the equation, $\boldsymbol{A}$ is the Langmuir current response constant, $\boldsymbol{B}$ is the adsorption constant of organics on the interface, and $\boldsymbol{C}$ is the polarization current (A).

From the result of fitting curve shown in Fig. 5a and the Langmuir adsorption equation, it is known that the
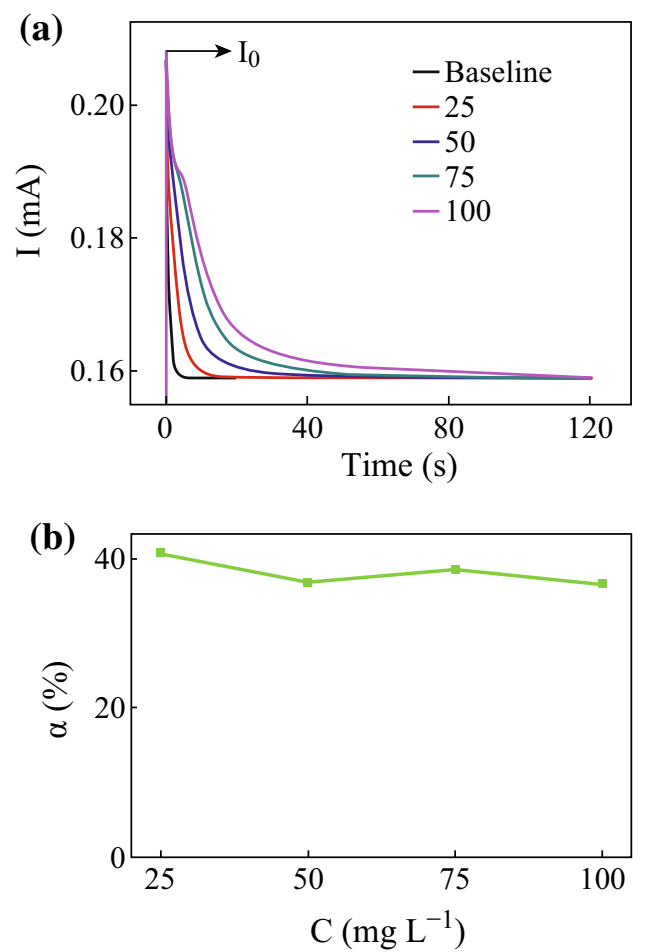

Fig. 4 a The response signals, and $\mathbf{b}$ the degree of the degradation of FA in different concentrations

adsorption constant of FA is 43.44. Based on the same method, the fitting curves of methanol, ethanediol, and glycerol shown in Fig. 5, could be obtained. Their adsorption constants were 19.32, 7.00, and 1.30, respectively. Thus, FA has the strongest adsorption property.

\subsection{The PEC Degradation Performance of FA}

To explore the PEC degradation performance of FA, different concentrations of FA $\left(25,50,75\right.$, and $\left.100 \mathrm{mg} \mathrm{L}^{-1}\right)$ 

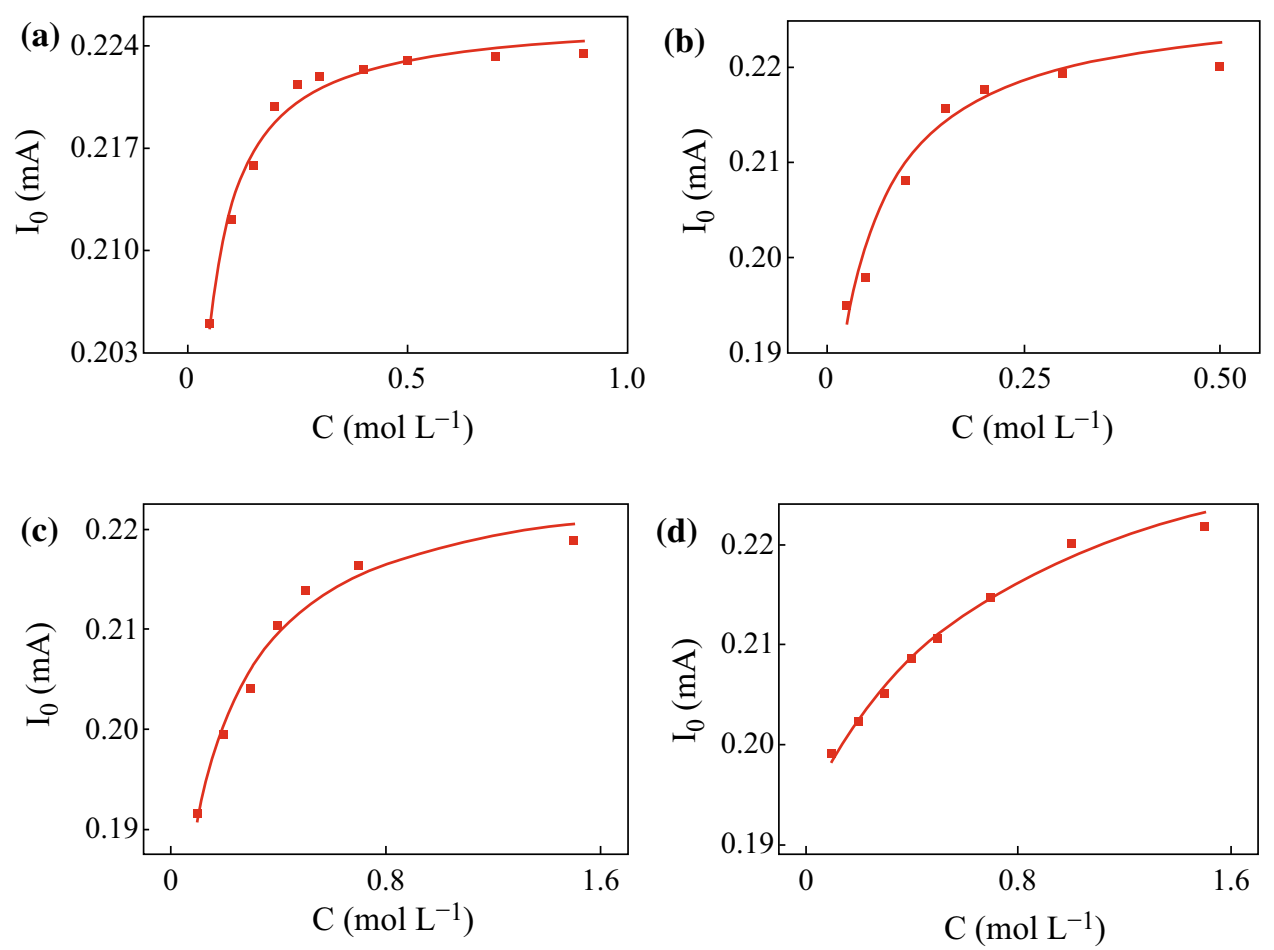

Fig. 5 The Langmuir fitting curves of a FA, b methanol, $\mathbf{c}$ ethanediol, and $\mathbf{d}$ glycerol

were degraded in the thin-layer reactor. The response signals obtained from the above degradation are shown in Fig. 3a. According to Eqs. 1 and 2, the degradation degree $(\alpha)$ can be calculated, showing the range from 36 to $41 \%$ as shown in Fig. 3b. Thus, FA could not be completely degraded alone in the PEC degradation.

The shapes of the $I-t$ curve in Fig. 3a are similar. Before the degradation reaction, a portion of FA molecular could be adsorbed on the surface of the TNAs electrode when the solution was injected into the reactor because of its strong adsorption property. Thus, the initial instantaneous current value is quite high, and it could rise as the concentration increases. Thereafter, the current value reduces rapidly and the degradation degree is less than $40 \%$. It can be deduced that the hydroxyl radicals, which can degrade FA, have been consumed mostly in the early stage of the reaction. This could result in the reduction of the reaction rate and a steady state in the end. For this reason, it is necessary to increase the amount of hydroxyl radicals which could make a complete PEC degradation of FA.

\subsection{The PEC Degradation Performance of Three LMHCs}

To explore the influence of LMHCs, the PEC degradation performance of the LMHCs is investigated. The $I-t$ curves in Fig. 6a show a similar shape by comparing the PEC degradation of glycerol in different concentrations. The current value declines a bit at the beginning, then increase for a short time (do not appear in low concentration, such as $50 \mathrm{mg} \mathrm{L}^{-1}$ ) and continuously declines until reaching a steady state. The increasing value represents the formation of the hydroxyl radicals from the degraded glycerol in the beginning of the PEC degradation, which could promote the degradation of the residual glycerol and the intermediate products. When most of glycerol is degraded, the promotion disappears and the current value reduces. According to Eq. 1, the degradation degree $(\alpha)$ of each concentration shown in Fig. $6 \mathrm{~b}$ could be calculated. Obviously, the degradation degree of glycerol with different concentrations is all close to $100 \%$. The degradation degree of both methanol and ethanediol shown in Fig. 6c, d is also about $100 \%$. Thus, it can be said that the three LMHCs could be degraded completely.

\subsection{The Promotion Effect of LMHCs and the Mechanism}

In this study, $50 \mathrm{mg} \mathrm{L}^{-1} \mathrm{FA}$ is chosen as the target organics and the three LMHCs with the concentrations of $50,100,150$, and $200 \mathrm{mg} \mathrm{L}^{-1}$ were added into the solution, respectively. Then the influence of each LMHC in the PEC degradation of FA was investigated.

Figure 7 shows the $I-t$ curves obtained from the PEC degradation of FA with addition of different concentrations of methanol. The integral area between two adjacent curves 

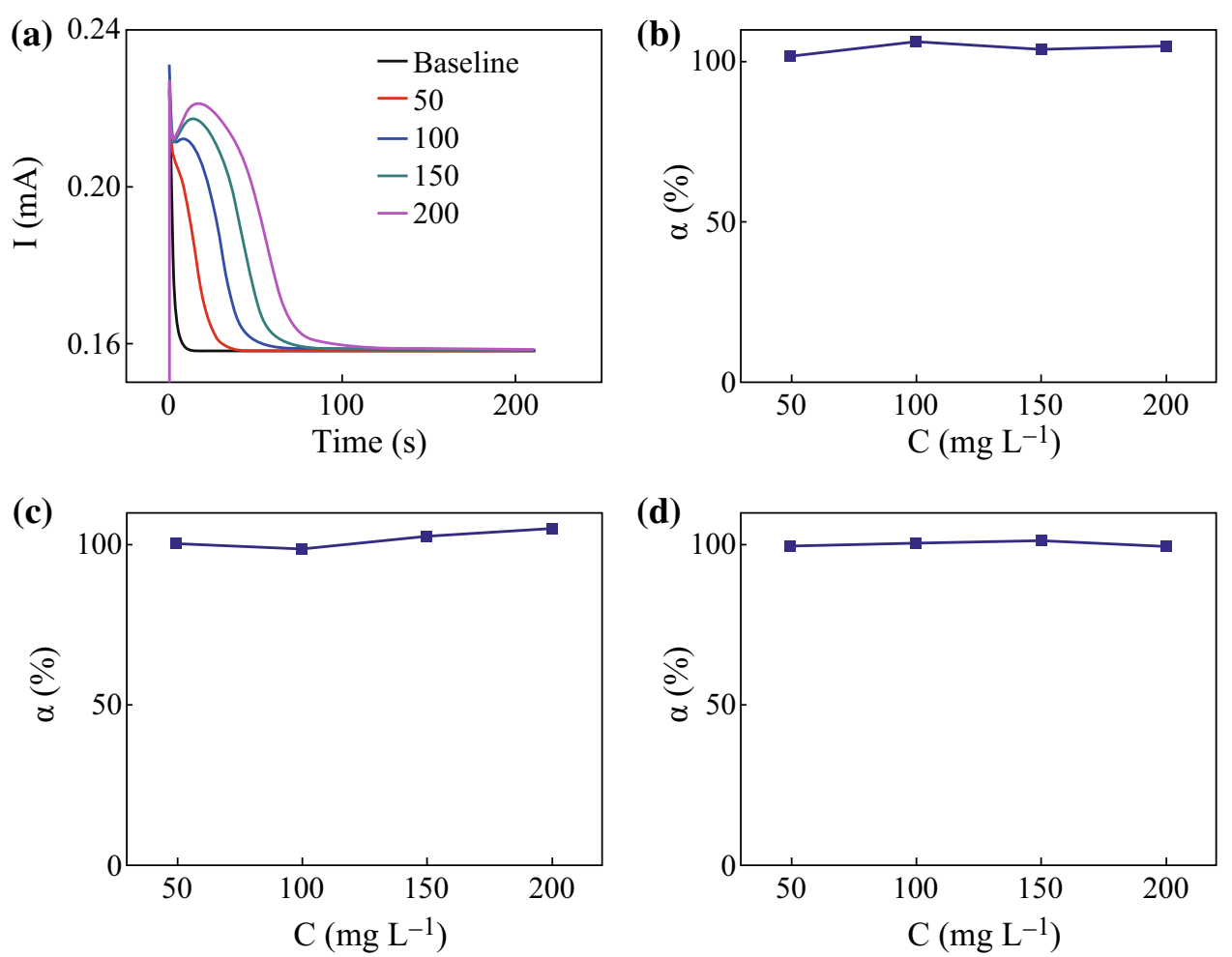

Fig. 6 a The response signals of glycerol's degradation. The degradation degree of three LMHCs in different concentrations: b glycerol, c methanol, and $\mathbf{d}$ ethanediol

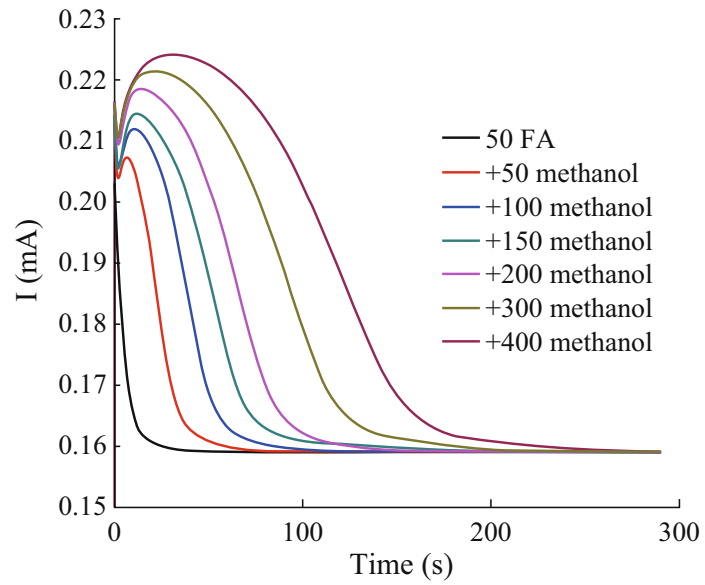

Fig. 7 The response signals obtained from the degradation of FA which is added methanol

is equivalent to the increment of methanol and further degraded FA. The amount of charge transfer generated by the degradation of FA could be obtained by reducing the $Q_{\text {th }}$ of added methanol from the $Q_{\text {net }}$ of mixture calculated from the $I-t$ curves according to the Eq. 2. According to the aforementioned, the three LMHCs could be degraded completely and their $Q_{\mathrm{th}}$ is represented by the quantity of transferred charges $\left(Q_{\text {net-glucose }}\right)$ generated in the exhausted PEC oxidation process of glucose in the same

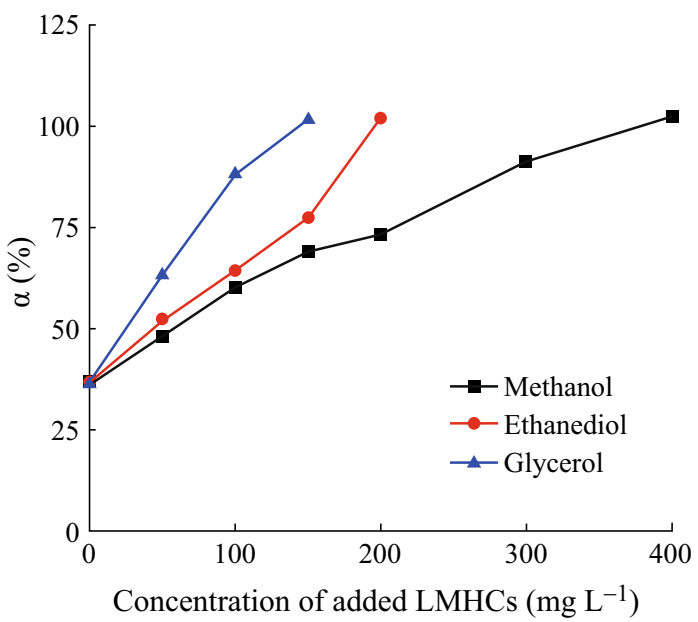

Fig. 8 The degradation degree of FA added three LMHCs

concentration. The each degradation degree of FA with addition of different concentrations of methanol could be calculated according to Eq. 1 .

The degradation degrees are shown in Fig. 8. As can be seen, methanol has an obvious promotion effect in the PEC degradation of FA. As the concentration of methanol increases, the degradation degree of FA rises. When $200 \mathrm{mg} \mathrm{L}^{-1}$ methanol was added, the degree is close to $80 \%$. After increasing the concentration of methanol, it 
Table 1 The degree and the concentration of hydroxyl in each $100 \mathrm{mg} \mathrm{L}^{-1}$ LMHCs

\begin{tabular}{lccc}
\hline $100 \mathrm{mg} \mathrm{L}^{-1}$ LMHCs & Methanol & Ethanediol & Glycerol \\
\hline Degree of degraded FA $(\%)$ & 60.38 & 64.49 & 88.35 \\
Hydroxyl concentration $\left(\mathrm{mol} \mathrm{L}^{-1}\right)$ & 2.0833 & 2.5 & 2.6786 \\
\hline
\end{tabular}

could be found that almost $400 \mathrm{mg} \mathrm{L}^{-1}$ methanol is needed to make sure that $50 \mathrm{mg} \mathrm{L}^{-1} \mathrm{FA}$ could be degraded completely. When different concentrations of ethanediol and glycerol are added, the degradation degrees of FA increase as well. Compared to the three LMHCs, glycerol shows the best promotion effect. To promote $50 \mathrm{mg} \mathrm{L}^{-1} \mathrm{FA}$ degraded completely, only $100 \mathrm{mg} \mathrm{L}^{-1}$ glycerol is needed rather than $200 \mathrm{mg} \mathrm{L}^{-1}$ ethanediol and $400 \mathrm{mg} \mathrm{L}^{-1}$ methanol. Meanwhile, when $100 \mathrm{mg} \mathrm{L}^{-1}$ of glycerol is added, the degradation degree of $50 \mathrm{mg} \mathrm{L}^{-1} \mathrm{FA}$ is much larger than the other conditions as shown in Table 1.

The mechanism of different LMHCs' promotion effect can be attributed to the difference of the hydroxyl's number in each molecule of the LMHCs. As shown in Table 1, the corresponding hydroxyl concentration that has $100 \mathrm{mg} \mathrm{L}^{-1}$ LMHCs influences the promotion effect. Obviously, glycerol provides the most hydroxyl radicals leading to the best promotion effect. Methanol molecule, which has only one hydroxyl, could not contribute enough hydroxyl radicals to promote the degradation. However, the difference of the adsorption constants could not influence their promotion effect. Although the adsorption of methanol is the highest, its promotion effect is not the best. It indicates that the amount of the hydroxyl in LMHCs makes a major effect.

\section{Conclusion}

In this study, a significant promotion effect of LMHCs, including methanol, ethanediol, and glycerol, was found in the PEC degradation of FA. The degradation degree of FA alone is just about $40 \%$, as the concentration of FA ranged from 25 to $100 \mathrm{mg} \mathrm{L}^{-1}$. When LMHCs are added, the degree increases obviously. Meanwhile, the promotion effects of methanol, ethanediol, and glycerol increase successively. Their influence mechanism is that the hydroxyls own influence their promotion effects. The more hydroxyls LMHC has, the better promotion effect it makes. This work provides a new way to promote the PEC degradation of FA and a direction to remove humus from polluted water.

Acknowledgments The authors would like to acknowledge the National High Technology Research and Development Program of China (Grant No. 2009AA063003) and the National Nature Science Foundation of China (Grant No.20677039) for financial support.

Open Access This article is distributed under the terms of the Creative Commons Attribution 4.0 International License (http://crea tivecommons.org/licenses/by/4.0/), which permits unrestricted use, distribution, and reproduction in any medium, provided you give appropriate credit to the original author(s) and the source, provide a link to the Creative Commons license, and indicate if changes were made.

\section{References}

1. H. Odegaard, B. Eikebrokk, R. Storhaug, Processes for the removal of humic substances from water: an overview based on norwegian experiences. Water Sci. Technol. 40(9), 37-46 (1999). doi:10.1016/S0273-1223(99)00638-1

2. B. Gao, Q. Yue, Natural organic matter (NOM) removal from surface water by coagulation. J. Environ. Sci. 17(1), 119-122 (2005)

3. Y. Wang, R. Mao, Q. Wang, Z. Yang, B. Gao, Y. Zhao, Fulvic acid removal performance and control of disinfection by-product formation potential in coagulation-ultrafiltration process. Desalination 302(20), 55-64 (2012). doi:10.1016/j.desal.2012.06.024

4. N.A. Klymenko, I.P. Kozyatnyk, L.A. Savchyna, Removing of fulvic acids by ozonation and biological active carbon filtration. Water Res. 44(18), 5316-5322 (2010). doi:10.1016/j.watres. 2010.05.035

5. S. Wang, X. Liu, W. Gong, W. Nie, B. Gao, Q. Yue, Adsorption of fulvic acids from aqueous solutions by carbon nanotubes. J. Chem. Technol. Biotechnol. 82(8), 698-704 (2007). doi:10. $1002 / j$ ctb. 1708

6. Y. Liu, H. Zhou, J. Li, H. Chen, D. Li, B. Zhou, W. Cai, Enhanced photoelectrochemical properties of $\mathrm{Cu}_{2} \mathrm{O}$-loaded short $\mathrm{TiO}_{2}$ nanotube array electrode prepared by sonoelectrochemical deposition. Nano-Micro Lett. 2(4), 277-284 (2010). doi:10.1007/ BF03353855

7. Y. Zhang, B. Tang, Z. Wu, H. Shi, Y. Zhang, G. Zhao, Glucose oxidation over ultrathin carbon-coated perovskite modified $\mathrm{TiO}_{2}$ nanotube photonic crystals with high-efficiency electron generation and transfer for photoelectrocatalytic hydrogen production. Green Chem. (2016). doi:10.1039/C5GC02745D (in press)

8. S. Sen Kavurmaci, M. Bekbolet, Tracing $\mathrm{TiO}_{2}$ photocatalytic degradation of humic acid in the presence of clay particles by excitation-emission matrix (EEM) fluorescence spectra. J. Photochem. Photobiol. A Chem. 282(15), 53-61 (2014). doi:10.1016/ j.jphotochem.2014.03.011

9. Q. Zhou, Y. Zhong, X. Chen, J. Liu, X. Huang, Y. Wu, Adsorption and photocatalysis removal of fulvic acid by $\mathrm{TiO}_{2}-$ graphene composites. J. Mater. Sci. 49(3), 1066-1075 (2013). doi:10.1007/s10853-013-7784-9

10. X. Cheng, H. Liu, Q. Chen, J. Li, X. Yu, Preparation and photoelectrocatalytic performance of $\mathrm{TiO}_{2}$ nano-tubes arrays electrode. Adv. Mater. Res. 661, 11-15 (2013). doi:10.4028/www. scientific.net/AMR.661.11

11. Q. Zheng, B. Zhou, J. Bai, L. Li, Z. Jin, J. Zhang, J. Li, Y. Liu, W. Cai, X. Zhu, Self-organized $\mathrm{TiO}_{2}$ nanotube array sensor for the determination of chemical oxygen demand. Adv. Mater. 20(5), 1044-1049 (2008). doi:10.1002/adma.200701619

12. X. Huangfu, Y. Wang, Y. Liu, X. Lu, X. Zhang, H. Cheng, J. Jiang, J. Ma, Effects of humic acid and surfactants on the aggregation kinetics of manganese dioxide colloids. Front. Environ. Sci. Eng. 9(1), 105-111 (2015). doi:10.1007/s11783-014-0726-1 
13. M. Boehme, W. Ensinger, Mixed phase anatase/rutile titanium dioxide nanotubes for enhanced photocatalytic degradation of methylene-blue. Nano-Micro Lett. 3(4), 236-241 (2011). doi:10. 1007/BF03353678

14. X. Li, Y. Jiang, W. Cheng, Y. Li, X. Xu, K. Lin, Mesoporous $\mathrm{TiO}_{2}$ /carbon beads: one-pot preparation and their application in visible-light-induced photodegradation. Nano-Micro Lett. 7(3), 243-254 (2015). doi:10.1007/s40820-015-0029-5

15. S. Song, J. Tu, Z. He, F. Hong, W. Liu, J. Chen, Visible lightdriven iodine-doped titanium dioxide nanotubes prepared by hydrothermal process and post-calcination. Appl. Catal. A Gen. 378(2), 169-174 (2010). doi:10.1016/j.apcata.2010.02.014

16. X. Li, H. Liu, P. Yue, Y. Sun, Photoelectrocatalytic oxidation of rose bengal in aqueous solution using a $\mathrm{Ti} / \mathrm{TiO}_{2}$ mesh electrode. Environ. Sci. Technol. 34(20), 4401-4406 (2000). doi:10.1021/ es000939k

17. Y. Zhang, G. Zhao, Y. Zhang, X. Huang, Highly efficient visiblelight-driven photoelectro-catalytic selective aerobic oxidation of biomass alcohols to aldehydes. Green Chem. 16(8), 3860-3869 (2014). doi:10.1039/C4GC00454J

18. J. Chang, C. Liu, J. Liu, Y. Zhou, X. Gao, S. Wang, Greenchemistry compatible approach to $\mathrm{Tio}_{2}$-supported $\mathrm{PdAu}$ bimetallic nanoparticles for solvent-free 1-phenylethanol oxidation under mild conditions. Nano-Micro Lett. 7(3), 307-315 (2015). doi:10.1007/s40820-015-0044-6

19. H. Wei, L. Wang, Z. Li, S. Ni, Q. Zhao, Synthesis and photocatalytic activity of one-dimensional $\mathrm{CdS} @ \mathrm{TiO}_{2}$ core-shell heterostructures. Nano-Micro Lett. 3(1), 6-11 (2011). doi:10. 1007/BF03353645

20. S. Song, Z. Liu, Z. He, A. Zhang, J. Chen, Y. Yang, X. Xu, Impacts of morphology and crystallite phases of titanium oxide on the catalytic ozonation of phenol. Environ. Sci. Technol. 44(10), 3913-3918 (2010). doi:10.1021/es100456n
21. Z. Liu, X. Zhang, S. Nishimoto, T. Murakami, A. Fujishima, Efficient photocatalytic degradation of gaseous acetaldehyde by highly ordered $\mathrm{TiO}_{2}$ nanotube arrays. Environ. Sci. Technol. 42(22), 8547-8551 (2008). doi:10.1021/es8016842

22. J. Staehelin, J. Hoigne, Decomposition of ozone in water in the presence of organic solutes acting as promoters and inhibitors of radical chain reactions. Environ. Sci. Technol. 19(12), 1206-1213 (1985). doi:10.1021/es00142a012

23. Q. Cai, M. Paulose, O.K. Varghese, C.A. Grimes, The effect of electrolyte composition on the fabrication of self-organized titanium oxide nanotube arrays by anodic oxidation. J. Mater. Res. 20(1), 230-236 (2011). doi:10.1557/JMR.2005.0020

24. X. Li, J. Li, J. Bai, Y. Dong, L. Li, B. Zhou, The inhibition effect of tert-butyl alcohol on the $\mathrm{TiO}_{2}$ nano assays photoelectrocatalytic degradation of different organics and its mechanism. NanoMicro Lett. (2016). doi:10.1007/s40820-015-0080-2

25. B. Liu, J. Li, B. Zhou, Q. Zheng, J. Bai, J. Zhang, Y. Liu, W. Cai, Kinetics and mechanisms for photoelectrochemical degradation of glucose on highly effective self-organized $\mathrm{TiO}_{2}$ nanotube arrays. Chin. J. Catal. 31(2), 163-170 (2010). doi:10.1016/S18722067(09)60042-5

26. Y. Su, G. Wang, D.T.F. Kuo, M. Chang, Y. Shih, Photoelectrocatalytic degradation of the antibiotic sulfamethoxazole using $\mathrm{TiO}_{2} / \mathrm{Ti}$ photoanode. Appl. Catal. B Environ. 186, 184-192 (2016). doi:10.1016/j.apcatb.2016.01.003

27. Y. Qin, M. Long, B. Tan, B. Zhou, RhB adsorption performance of magnetic adsorbent $\mathrm{Fe}_{3} \mathrm{O}_{4} / \mathrm{RGO}$ composite and its regeneration through a fenton-like reaction. Nano-Micro Lett. 6(2), 125-135 (2014). doi:10.1007/BF03353776

28. N. Talebian, M.R. Nilforoushan, Comparative study of the structural, optical and photocatalytic properties of semiconductor metal oxides toward degradation of methylene blue. Thin Solid Films 518(8), 2210-2215 (2010). doi:10.1016/j.tsf.2009.07.135 